\title{
Magnetic-resonance-guided Radiation Therapy With Simultaneous Integrated Boost at Mid-bladder Volume for Bladder Cancer
}

Janjira Petsuksiri ( $\square$ janjira102@yahoo.com )

Mahidol University Faculty of Medicine Siriraj Hospital https://orcid.org/0000-0002-2334-4899

Chanida Sathitwatthanawirot

Mahidol University Faculty of Medicine Siriraj Hospital

Utumporn Puangragsa

Mahidol University Faculty of Medicine Siriraj Hospital

Wisawa Phongprapun

Mahidol University Faculty of Medicine Siriraj Hospital

Pittaya Dankulchai

Mahidol University Faculty of Medicine Siriraj Hospital

Nantakan Apiwarodom

Mahidol University Faculty of Medicine Siriraj Hospital

\section{Case report}

Keywords: Bladder cancer, MRgRT, MR-guided radiation therapy, SIB, Simultaneous integrated boost

Posted Date: August 3rd, 2021

DOl: https://doi.org/10.21203/rs.3.rs-751077/v1

License: (9) (i) This work is licensed under a Creative Commons Attribution 4.0 International License. Read Full License 


\section{Abstract}

Background and Purpose: To report the workflow and dose accumulation for bladder preservation for a bladder cancer patient, using magnetic-resonance-guided radiation therapy (MRgRT) and the simultaneous integrated boost (SIB) technique at mid-bladder volume.

Materials and Methods: A muscle-invasive bladder cancer patient was treated with MRgRT. The patient was treated with the SIB technique at mid-bladder volume, with $45 \mathrm{~Gy}$ to the whole bladder (CTV $\left.{ }_{\text {WB }}\right)$ and $55 \mathrm{~Gy}$ to the tumor bed (CTV boost $)$ in 20 fractions. Daily re-optimization with an adapt-to-position (ATP) strategy was utilized for dose adjustment to encompass the bladder within anisotropic planning target volume (PTV ${ }_{W B}$ and PTV boost).

Results: The mean daily treatment time was 55 minutes (range, 35-73). The actual whole-bladder and tumor-bed-boost doses were $45.74 \pm 5.91$ and $54.1 \pm 4.62 \mathrm{~Gy}$, respectively. PTV ${ }_{W B}$ encompassing $C T V_{W B}$ was $95.69 \% \pm 5.36 \%$. PTV boost encompassing CTV boost was $97.52 \% \pm 6.05 \%$. The actual rectal and bowel doses were below the reference plan doses.

Conclusions: The use of MRgRT with the SIB and ATP strategy proved feasible for bladder cancer treatment. Mid-bladder volume allowed treatment with the SIB technique under MR monitoring.

\section{Background And Purposes}

The current standard for bladder-preservation treatment for muscle-invasive bladder cancer (MIBC) is a multimodal approach, comprising transurethral resection of the bladder tumor (TUR-BT), chemotherapy, and radiation therapy (1-3). Achieving acceptable target coverage during radiation therapy for bladder cancer is challenging due to the filling of the bladder during treatment, causing both intrafraction and interfraction organ motion (4-6). Magnetic-resonance-guided radiation therapy (MRgRT) offers the opportunity to adapt and re-optimize radiation doses at each fraction, thereby ensuring that they are compatible with anatomical changes at each treatment. This study reports the feasibility of MRgRT, using the simultaneous integrated boost (SIB) technique at mid-bladder volume with adapt to position (ATP) strategy for bladder-preservation treatment.

\section{Materials And Methods}

\section{A. Patient data}

An 89-year-old female patient with multiple medical comorbidities was diagnosed with a high-grade, urothelial carcinoma, clinical stage T2NOM0, post TUR-BT. Given her elderly age and comorbidities, she was not a candidate for radical surgery. She opted to receive concurrent chemoradiation (weekly carboplatin) for bladder preservation with a curative aim. Inform consent was obtained from the patient for this publication. 


\section{B. Treatment planning}

The patient was simulated with an empty bladder and empty rectum. Intravenous contrast-enhanced CT scanning was performed at $0,15,30$, and 45 minutes after contrast injection, thereby providing 4 sets of CT images. The CT scan at 15 minutes (CT plan 15 mins) was selected for contouring and treatment planning. It was based on the midpoint of the whole bladder volume obtained from the four data sets. The clinical target volume-whole bladder (CTV ${ }_{\mathrm{WB}}$ ) encompassed the whole bladder with $1 \mathrm{~cm}$ of the surrounding soft tissue for microscopic extension. The anisotropic planning target volume-WB (PTV $\left.{ }_{W B}\right)$ was determined by adding $5 \mathrm{~mm}$ laterally and inferiorly, $1.5 \mathrm{~cm}$ anteriorly and superiorly, and $1 \mathrm{~cm}$ posteriorly (7). The CTV-tumor bed (CTV boost) encompassed the whole right lateral bladder wall with a 1-cm margin, except $1.5 \mathrm{~cm}$ medially. The PTV-tumor bed (PTV boost) was established by adding $5 \mathrm{~mm}$ in all directions of the CTV boost $_{\text {. The PTV }}$ WB $_{\text {and PTV }}$ boost encompassed the whole bladder volume and the right lateral wall consecutively, for each $C T$ data set $(0,15,30$, and 45 minutes). The rectum and small bowel were contoured at CT plan 15 mins. The PTV ${ }_{\text {WB }}$ and PTV boost were prescribed with $45 \mathrm{~Gy}$ and $55 \mathrm{~Gy}$ for a total of 20 fractions with an SIB schedule (8).

Planning was performed using Monaco Unity radiation treatment planning software (version 5.40.01; Elekta Inc., Saint Charles, MO, USA). An intensity-modulated radiation therapy with 7 beam angles $(0,30$, $160,200,240,270$, and 320 degrees) was generated using the CT plan 15 mins data. The grid spacing was $0.3 \mathrm{~cm}$, with a $1 \%$ statistical uncertainty. There were 150 maximal segments with a 0.5 -cm segment width; a 2-cm² maximal segment area; 4 monitor units per segment; and 15 sub second-pulse loops.

The dose constraints (9) and planned dose delivery (based on CT plan 15 mins) for each organ was detailed in Supplementary Table 1. The reference plan illustrated in Supplementary Fig. 1.

\section{Online adaptive workflow}

On a daily basis, the patient's rectum and bladder were emptied prior to set up. The workflow is depicted in Supplementary Fig. 2. A T2-weighted MR scan (MRI T2) was acquired for treatment planning purposes. The MRI T2 was fused to CT plan 15 mins by using pelvic bone rigid fusion. For treatment planning, we selected adapt-to-position (ATP) with optimized shape strategies. The daily treatment doses were adjusted to achieve the CTV target doses.

After treatment plan approval, bladder volume monitoring was performed with MR motion monitoring (MM). Treatment commenced when the bladder volume during the MM reached the bladder volume specified in $\mathrm{CT}$ plan 15 mins $\left(C T V_{W B}\right)$. The MR T2 images that were delivered immediately after starting the first radiation beams $\left(\mathrm{MR}_{\text {first }}\right)$ and before the last radiation beams $\left(M R_{\text {last }}\right)$ were employed to represent the first and last treatment volumes, respectively. PTV ${ }_{W B}$ was used for target monitoring during the MM, with PTV ${ }_{W B}$ encompassing the whole urinary bladder during treatment. Treatment was stopped if the bladder volume during the MM extended outside PTV ${ }_{\text {WB }}$. CTV ${ }_{\text {boost }}$ was monitored on the mid-axial and 
coronal scans during the MM. The beam-on time was approximately 15 mins. Figure 1 shows $C T V_{W B}$ and PTV ${ }_{W B}$ on $C T$ plan 15 mins, $M M$ at the beam starting point, and $M M$ at the end of treatment.

The CTV ${ }_{\text {WB }}$ and CTV boost for the rectum and bowel were re-contoured by single physician (JP) for each daily $\mathrm{MR}_{\text {first }}$ and MRI last, to recalculate the accumulated radiation doses delivered to the target and normal organs.

\section{Results}

On a daily basis, a total of 19 MRgRTs were delivered to the patient via an Elekta Unity system. Due to machine downtime, one additional treatment was delivered using an Elekta Versa HD linear accelerator with cone beam CT verification. The mean daily time for the whole workflow-from the MR survey to the end of treatment-was 55 minutes (range, 35-73) [Supplementary Table 3].

The actual cumulative doses delivered, by organ were demonstrated in Supplementary Table 1. As to dose accumulation, the CTV boost and CTV ${ }_{\text {WB }}$ were recontoured on $\mathrm{MRI}_{\text {first }}$ and MRI last. The ratio of CTV boost to $C T V_{W B}$ was maintained in the range of 0.32 to 0.39 , consistent with the ratio of CTV boost to CTV ${ }_{W B}$ in the reference plan (0.37). The actual cumulative doses delivered from the MRgRT-CT plan registration are presented in Table 1. The actual average doses (D 95) delivered to CTV boost and CTV WB $_{\text {B }}$ were $54.1 \pm 4.62$ Gy and $45.74 \pm 5.91$, respectively. The rectum and bowel dose deliveries were below the respective reference plan doses (Supplementary Table 2). Figure 2 presents the dose-volume histogram (DVH) data of each MRgRT-CT plan 15 mins registration for CTV $_{\text {boost }}, \mathrm{CTV}_{\mathrm{WB}}$, rectum, and bowel. 
Table 1

Actual dose delivered for each target on MR T2 during the treatments

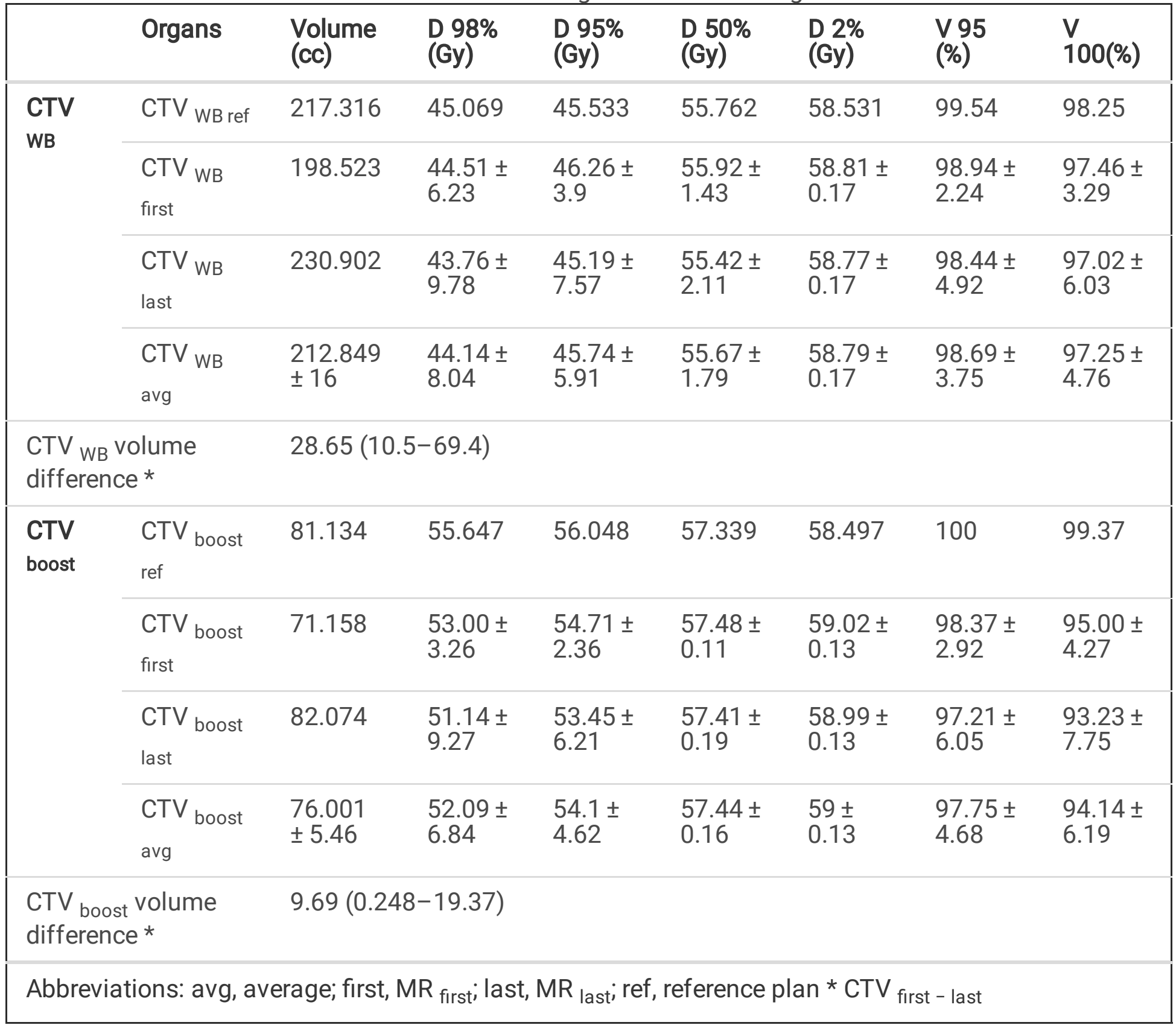

During the treatments, PTV ${ }_{\text {WB }}$ encompassing CTV ${ }_{\text {WB }}$ was $95.69 \% \pm 5.36 \%\left(96.89 \% \pm 3.54 \%\right.$ for MR $_{\text {first }}$; $94.5 \% \pm 6.49 \%$ for $\left.\mathrm{MR}_{\text {last }}\right)$. PTV boost encompassing CTV $_{\text {boost }}$ was $97.52 \% \pm 6.05 \%(98.15 \% \pm 3.83 \%$ for $M R_{\text {first }} ; 96.89 \% \pm 7.6 \%$ for $\left.\mathrm{MR}_{\text {last }}\right)$.

The patient tolerated the chemoradiation well. She had no genitourinary tract or gastrointestinal tract complications during her course of radiation therapy. Three months after the chemoradiation therapy, the patient underwent a follow-up cystoscopy; it did not detect any gross residual tumor on the right lateral wall. Moreover, the patient did not report any urinary symptoms or gastrointestinal side effects following the therapy. 


\section{Discussion}

The radiation therapy is a critical component of bladder-preservation treatment (1-3). The goals of radiation therapy are to enhance bladder preservation rates while minimizing the associated toxicities. In terms of dose fractionation, a hypofractionated radiation therapy schedule ( $55 \mathrm{~Gy}$ in 20 fractions) has been recognized as equivalent to conventional fractionation with sequential boost (64-70 Gy in 32-35 fractions) for locoregional control in an individual patient data meta-analysis (8). In addition, the optimal volume of radiation treatment is controversial. The rationale for not treating pelvic lymph nodes is to improve the tolerability of the therapy by excluding normal tissue from the treatment area while achieving an acceptable rate of nodal failure (4.9\%) (10). Another issue of radiation treatment is whether to irradiate part of the bladder or the whole bladder. A randomized trial reported that only $7 \%$ of tumor recurrences were located outside the irradiated volume for patients who underwent a partial bladder irradiation (11, 12). However, these studies were planned with an empty bladder, in which the gross tumor volume delineation is potentially inaccurate. Furthermore, given the generous isotropic margins with an empty bladder, planning with a 3D conformal technique would rarely spare an unaffected urinary bladder. As a result, these studies failed to decrease the treatment toxicities by using partial bladder irradiation.

Based on these rationales, we decided to treat only the urinary bladder with a hypofractionated radiation schedule. The plan was generated using a mid-bladder volume and the SIB technique to administering a high dose to the tumor bed area with MR monitoring during the treatments.

Importantly, treating bladder cancer is made difficult by the uncertainties of bladder position, shape, and volume during treatment. These stem from the wide variations in nonuniform expansion caused by bladder filling, impacting on both intrafraction and interfraction motion. Research has been conducted to determine the adequacy of target coverage during treatment. Individual urinary-flow rates, bladder-shapechange models, and treatment times have been utilized to generate libraries of accurate treatment volumes $(4,6)$. Nevertheless, the library plans may not encompass the bladder dimensions on some specific days $(13,14)$.

MRgRT is an online adaptive tool that enable radiation treatment plans to be adjusted in accordance with actual patient anatomy on each treatment day. Not only does MRgRT ensure target coverage, but it permits doses to the targets and organs at risk to be re-optimized using either an (adapt-to-shape) ATS or (adapt-to-position) ATP strategy (15).

Hunt et al. reported that bladder cancer treatments with MRgRT could achieve a $96.6 \%$ target coverage (7). They reported a workflow that started with an empty bladder, anisotropic margins, and ATS reoptimization. However, $14 \%$ of their patients need re-optimization with ATP, given the alterations to the bladder shapes and volumes between the image acquisition and the treatment starting time. In addition, the treatments were planned with an empty bladder. This approach did not permit the use of the SIB technique to restrict the application of high-dose volumes to the affected sites only. 
In our study, we elected to use the mid-bladder volume, with the aim of prescribing a high dose to the tumor bed and a microscopic dose to the uninvolved bladder. We generated an individual anisotropic PTV margins around the mid-bladder volume from serial images on CT simulation to encompass the target volumes until the end of treatment. We chose to re-optimize with ATP to shorten the treatment planning process to account for patient tolerability of full bladder. As a result, we were able to use this strategy throughout the whole treatment, achieving more than a 96-98\% target coverage for both CTV ${ }_{\text {WB }}$ and

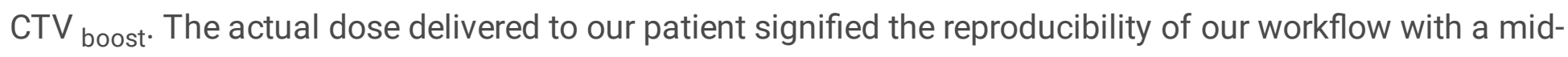
bladder protocol.

Essentially, patient preparation for hydration status and rectal contents was crucial. We recommend the patient to have consistent fluid intake before the scheduled treatment sessions to stabilize the bladder volume during treatment. The use of a low-fiber diet and laxatives is also recommended to help the patient achieve an empty rectum before each treatment session. These approaches decrease the uncertainties associated with the bowel and rectum adjacent to the bladder.

The strength of this study is the reproducible and successful strategy of mid-bladder volume and the SIB technique to decrease excessive radiation doses to the whole urinary bladder. In addition, bladder preparation protocol with personalized anisotropic PTV enhances the success of the ATP approach to shorten the length of the workflow.

Nevertheless, the workflow we have described has several limitations. For one thing, the recontouring

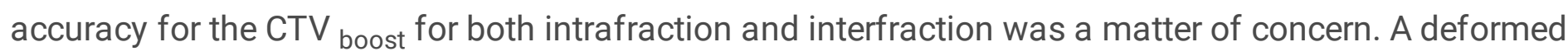
contour propagation may minimize these uncertainties. As to the dose delivered on a daily basis, dose adjustment was employed mainly for the target coverage (CTV). Therefore, the surrounding normal structures may receive different doses from the reference plan. With ATP, the tissue densities from the initial CT simulation were used for the dose calculation. Therefore, the different soft tissue densities on each specific day resulting from organ motion may not be represented in the actual dose delivered.

\section{Conclusions}

Bladder preservation for MIBC using the mid-bladder volume and the SIB technique was feasible. MRgRT with ATP re-optimization assisted this approach by obtaining proper target coverage and safety for the surrounding normal tissues. Applying the mid-bladder volume with adequate individual PTV margins proved to be a feasible protocol to counter the variations attributable to urinary filling over the course of the treatments.

\section{List Of Abbreviations}

MIBC: muscle-invasive bladder cancer

TUR-BT: transurethral resection of the bladder tumor 
MRgRT: Magnetic-resonance-guided radiation therapy

MRI T2: T2-weighted MR scan

MM: MR motion monitoring

$M R_{\text {first }}:$ MR T2 images immediately after starting the first radiation beams

$M R_{\text {last }}:$ MR T2 images before the last radiation beams

SIB: simultaneous integrated boost

CTV ${ }_{\text {WB}}$ : clinical target volume-whole bladder

CTV $\mathrm{boost}_{\text {: }}$ clinical target volume -tumor bed

PTV ${ }_{\text {WB}}$ : planning target volume- whole bladder

PTV ${ }_{\text {boost }}$ : planning target volume- tumor bed

ATP: adapt-to-position

ATS: adapt-to-shape

\section{Declarations}

\section{Ethics approval and consent to participate}

Inform consent was obtained from the patient for data evaluation and publication.

Consent for publication

Inform consent was obtained from the patient for this publication.

\section{Availability of data and materials}

All data generated or analyzed during this study are included in this published article and its additional files.

\section{Competing interests}

Department of Radiation Oncology Faculty of Medicine Siriraj Hospital is a member of the Elekta MRLinac Consortium. No commercial financial support was received from any organization for this work. The authors has no conflicts to disclose.

\section{Funding}


The Faculty of Medicine Siriraj Hospital, Mahidol University, funded this publication.

\section{Authors 'contributions}

All authors (JP, CS, UP, WP, PD, and NA) were involved in patient management, data collection and evaluation. JP wrote the draft of this manuscript. All authors contributed to edit this manuscript.

\section{Acknowledgements}

The authors thank Professor Imjai Chitapanarux, Kullathorn Thephamongkhol and Jiraporn Setakornnukul for comments and suggestions on this manuscript. We thank Mr.David Park for English editing.

\section{References}

1. Chang SS, Bochner BH, Chou R, Dreicer R, Kamat AM, Lerner SP, et al. Treatment of Non-Metastatic Muscle-Invasive Bladder Cancer: AUA/ASCO/ASTRO/SUO Guideline. J Urol 2017;198:552-9.

2. Garcia-Perdomo HA, Montes-Cardona CE, Guacheta M, Castillo DF, Reis LO. Muscle-invasive bladder cancer organ-preserving therapy: systematic review and meta-analysis. World J Urol 2018;36:19972008.

3. Royce TJ, Feldman AS, Mossanen M, Yang JC, Shipley WU, Pandharipande PV, et al. Comparative Effectiveness of Bladder-preserving Tri-modality Therapy Versus Radical Cystectomy for Muscleinvasive Bladder Cancer. Clin Genitourin Cancer 2019;17:23-31 e3.

4. Lotz HT, van Herk M, Betgen A, Pos F, Lebesque JV, Remeijer P. Reproducibility of the bladder shape and bladder shape changes during filling. Med Phys 2005;32:2590-7.

5. Kibrom AZ, Knight KA. Adaptive radiation therapy for bladder cancer: a review of adaptive techniques used in clinical practice. J Med Radiat Sci 2015;62:277-85.

6. Dees-Ribbers HM, Betgen A, Pos FJ, Witteveen T, Remeijer P, van Herk M. Inter- and intra-fractional bladder motion during radiotherapy for bladder cancer: a comparison of full and empty bladders. Radiother Oncol 2014;113:254-9.

7. Hunt A, Hanson I, Dunlop A, Barnes H, Bower L, Chick J, et al. Feasibility of magnetic resonance guided radiotherapy for the treatment of bladder cancer. Clin Transl Radiat Oncol 2020;25:46-51.

8. Choudhury A, Porta N, Hall E, Song YP, Owen R, MacKay R, et al. Hypofractionated radiotherapy in locally advanced bladder cancer: an individual patient data meta-analysis of the BC2001 and BCON trials. Lancet Oncol 2021;22:246-55.

9. Dearnaley D, Syndikus I, Mossop H, Khoo V, Birtle A, Bloomfield D, et al. Conventional versus hypofractionated high-dose intensity-modulated radiotherapy for prostate cancer: 5-year outcomes of the randomised, non-inferiority, phase 3 CHHiP trial. Lancet Oncol 2016;17:1047-60.

10. James ND, Hussain SA, Hall E, Jenkins $P$, Tremlett J, Rawlings $C$, et al. Radiotherapy with or without chemotherapy in muscle-invasive bladder cancer. N Engl J Med 2012;366:1477-88. 
11. Cowan RA, McBain CA, Ryder WD, Wylie JP, Logue JP, Turner SL, et al. Radiotherapy for muscleinvasive carcinoma of the bladder: results of a randomized trial comparing conventional whole bladder with dose-escalated partial bladder radiotherapy. Int J Radiat Oncol Biol Phys 2004;59:197207.

12. Huddart RA, Hall E, Hussain SA, Jenkins P, Rawlings $C$, Tremlett J, et al. Randomized noninferiority trial of reduced high-dose volume versus standard volume radiation therapy for muscle-invasive bladder cancer: results of the BC2001 trial (CRUK/01/004). Int J Radiat Oncol Biol Phys 2013;87:2619.

13. Foroudi F, Pham D, Rolfo A, Bressel M, Tang Cl, Tan A, et al. The outcome of a multi-centre feasibility study of online adaptive radiotherapy for muscle-invasive bladder cancer TROG 10.01 BOLART. Radiother Oncol 2014;111:316-20.

14. Hafeez S, Warren-Oseni K, McNair HA, Hansen VN, Jones K, Tan M, et al. Prospective Study Delivering Simultaneous Integrated High-dose Tumor Boost ( $</=70 \mathrm{~Gy})$ With Image Guided Adaptive Radiation Therapy for Radical Treatment of Localized Muscle-Invasive Bladder Cancer. Int J Radiat Oncol Biol Phys 2016;94:1022-30.

15. Vestergaard A, Hafeez S, Muren LP, Nill S, Hoyer M, Hansen VN, et al. The potential of MRI-guided online adaptive re-optimisation in radiotherapy of urinary bladder cancer. Radiother Oncol 2016;118:154-9.

\section{Figures}

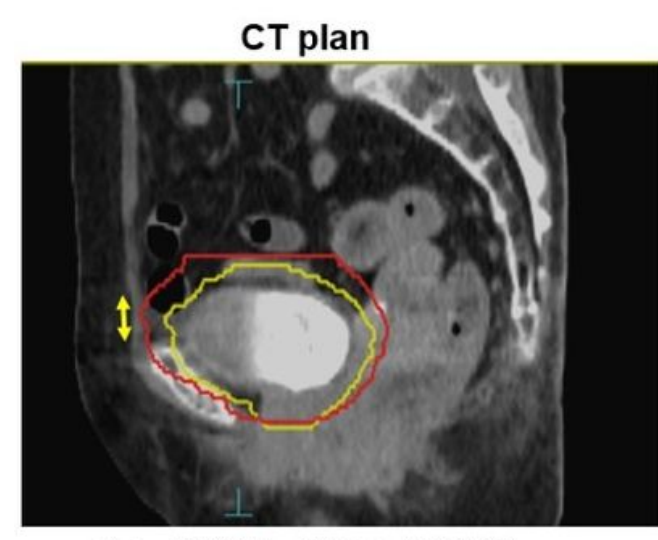

Red - PTV WB, Yellow-CTV WB
Start treatment

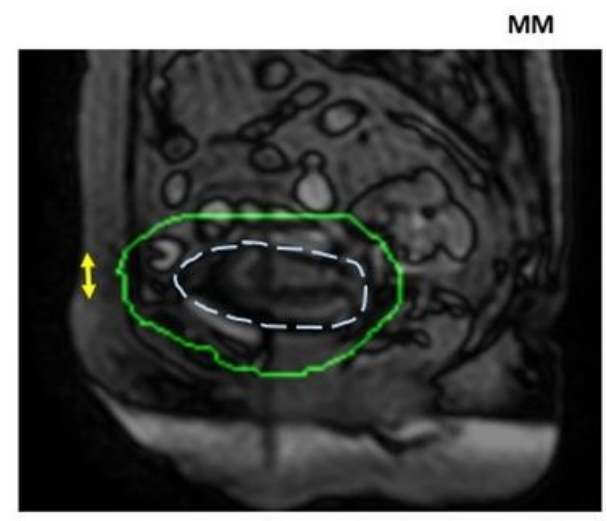

Green - PTV WB ,
End of treatment

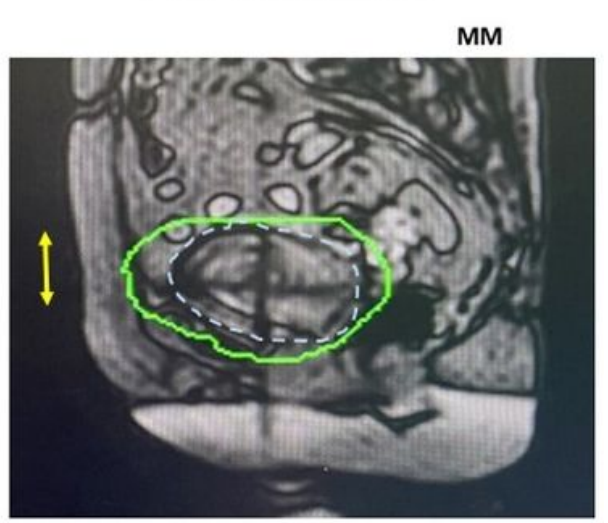

Light blue - bladder outline during treatment

Figure 1

CT plan 15 mins (left) and motion monitoring (MM) at the start (middle) and at the end (right) of the treatments. 

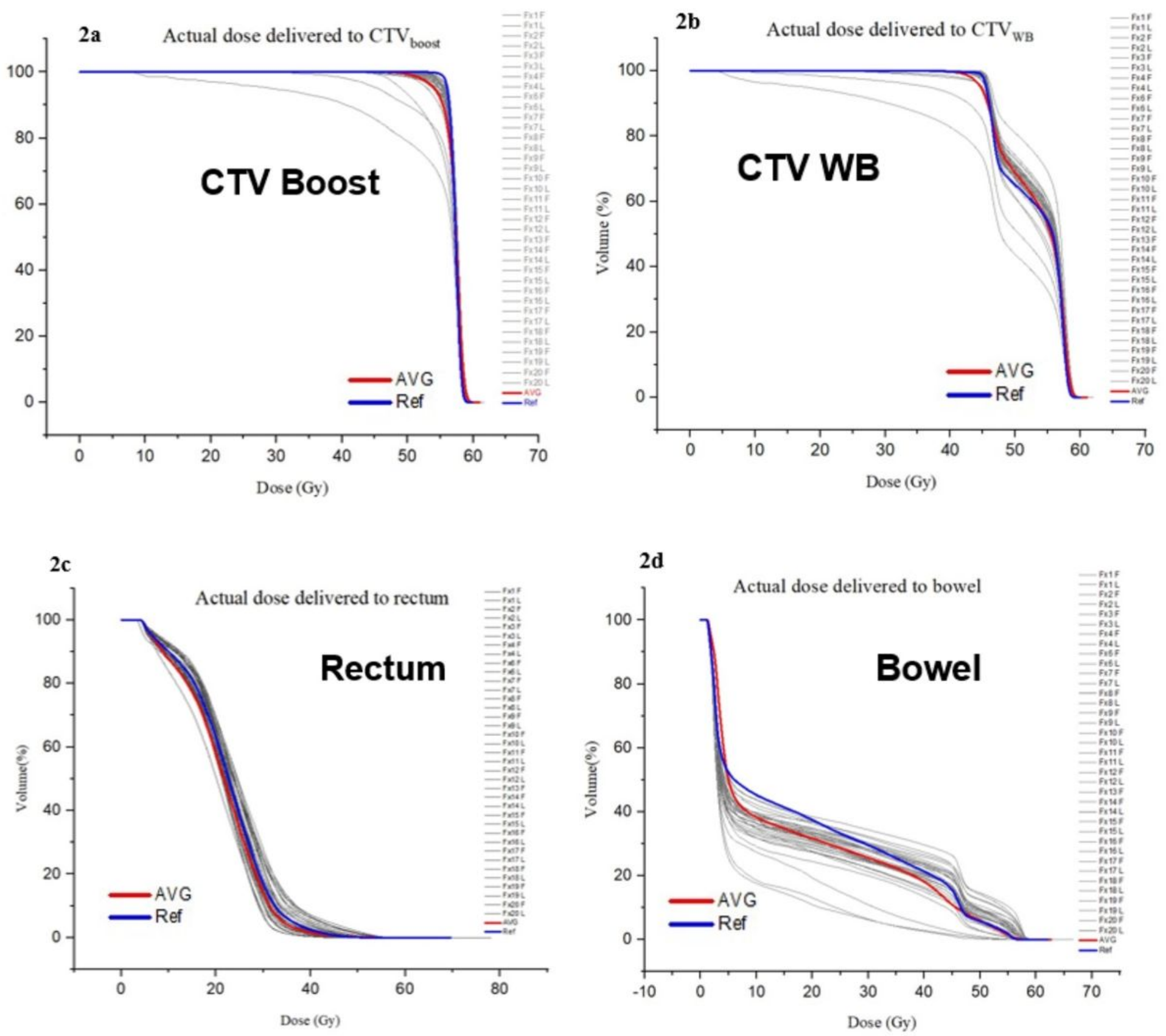

Figure 2

Dose-volume histogram (DVH) data of each MRgRT-CT plan 15 mins registration for CTV boost (2a), CTV WB $(2 b)$, rectum $(2 c)$, and bowel $(2 d)$.

\section{Supplementary Files}

This is a list of supplementary files associated with this preprint. Click to download.

- Additionalfile1SuppTables13.docx

- Additionalfile2SupplementaryFigures12.docx 